\title{
Tight Bounds for Lp Samplers, Finding Duplicates in Streams, and Related Problems
}

\author{
Hossein Jowhari \\ Simon Fraser University \\ School of Computing Science \\ Burnaby, BC, Canada \\ hjowhari@cs.sfu.ca
}

\author{
Mert Sağlam \\ Simon Fraser University \\ School of Computing Science \\ Burnaby, BC, Canada \\ mert.saglam@sfu.ca
}

\author{
Gábor Tardos \\ Rényi Institute of Mathematics \\ Budapest, Hungary \\ and \\ Simon Fraser University \\ School of Computing Science \\ Burnaby, BC, Canada \\ tardos@renyi.hu
}

\begin{abstract}
In this paper, we present near-optimal space bounds for $L_{p^{-}}$ samplers. Given a stream of updates (additions and subtraction) to the coordinates of an underlying vector $x \in$ $\mathbb{R}^{n}$, a perfect $L_{p}$ sampler outputs the $i$-th coordinate with probability $\left|x_{i}\right|^{p} /\|x\|_{p}^{p}$. In SODA 2010, Monemizadeh and Woodruff showed polylog space upper bounds for approximate $L_{p}$-samplers and demonstrated various applications of them. Very recently, Andoni, Krauthgamer and Onak improved the upper bounds and gave a $O\left(\epsilon^{-p} \log ^{3} n\right)$ space $\epsilon$ relative error and constant failure rate $L_{p}$-sampler for $p \in$ $[1,2]$. In this work, we give another such algorithm requiring only $O\left(\epsilon^{-p} \log ^{2} n\right)$ space for $p \in(1,2)$. For $p \in(0,1)$, our space bound is $O\left(\epsilon^{-1} \log ^{2} n\right)$, while for the $p=1$ case we have an $O\left(\log (1 / \epsilon) \epsilon^{-1} \log ^{2} n\right)$ space algorithm. We also give a $O\left(\log ^{2} n\right)$ bits zero relative error $L_{0}$-sampler, improving the $O\left(\log ^{3} n\right)$ bits algorithm due to Frahling, Indyk and Sohler.

As an application of our samplers, we give better upper bounds for the problem of finding duplicates in data streams. In case the length of the stream is longer than the alphabet size, $L_{1}$ sampling gives us an $O\left(\log ^{2} n\right)$ space algorithm, thus improving the previous $O\left(\log ^{3} n\right)$ bound due to Gopalan and Radhakrishnan.

In the second part of our work, we prove an $\Omega\left(\log ^{2} n\right)$ lower bound for sampling from $0, \pm 1$ vectors (in this special case, the parameter $p$ is not relevant for $L_{p}$ sampling). This matches the space of our sampling algorithms for constant $\epsilon>0$. We also prove tight space lower bounds for the finding duplicates and heavy hitters problems. We obtain these lower bounds using reductions from the communication complexity problem augmented indexing.
\end{abstract}

\footnotetext{
* Supported by NSERC grant 329527, OTKA grants T046234, AT-048826, and NK-62321
}

Permission to make digital or hard copies of all or part of this work for personal or classroom use is granted without fee provided that copies are not made or distributed for profit or commercial advantage and that copies bear this notice and the full citation on the first page. To copy otherwise, to republish, to post on servers or to redistribute to lists, requires prior specific permission and/or a fee.

PODS'11, June 13-15, 2011, Athens, Greece.

Copyright 2011 ACM 978-1-4503-0660-7/11/06 ...\$10.00.

\section{Categories and Subject Descriptors}

F.2.0 [Analysis of Algorithms and Problem Complexity]: General; E.4 [Coding and Information Theory]: Formal models of communication; E.m [Data]: Miscellaneous

\section{General Terms}

Algorithm, Design, Theory

\section{Keywords}

Streaming Algorithms, Sampling, Finding Duplicates

\section{INTRODUCTION}

Sampling has become an indispensable tool in analysing massive data sets, and particularly in processing data streams. In the past decade, several sampling techniques have been proposed and studied for the data stream model $[3,11,5,10$, $22,1]$. In this work, we study $L_{p}$-samplers, a new variant of space efficient samplers for data streams that was introduced by Monemizadeh and Woodruff in [22]. Roughly speaking, given a stream of updates (additions and subtraction) to the coordinates of an underlying vector $x \in \mathbb{R}^{n}$, an $L_{p}$-sampler processes the stream and outputs a sample coordinate of $x$ where the $i$-th coordinate is picked with probability proportional to $\left|x_{i}\right|^{p}$.

In [22], it was observed that $L_{p^{-}}$samplers lead to alternative algorithms for many known streaming problems, including heavy hitters and frequency moment estimation. Here in this paper, we focus on a specific application, namely finding duplicates in long streams; although our $L_{p}$ samplers work and often give better space performance for all applications listed in [22]. We refer the reader to [22] and [1] for further applications of $L_{p}$-samplers.

Observe that we allow both negative and positive updates to the coordinates of the underlying vector. In the case where only positive updates are allowed and $p=1$, the problem is well understood. For instance the classical reservoir sampling [19] from the 60's (attributed to Alan G. Waterman) gives a simple solution as follows. Given a pair $(i, u)$, indicating an addition of $u$ to the $i$-th coordinate of the underlying vector $x$, the sampler having maintained $s$, the sum of the updates seen so far, replaces the current sample with $i$ with probability $u / s$, otherwise does nothing and moves to 
the next update. It is easy to verify that this is a perfect $L_{1}$-sampler and the space usage is only $O(1)$ words.

With the presence of negative updates, sampling becomes a non-trivial problem. In this case, it is not clear at all how to maintain samples without keeping track of the updates to the individual coordinates. In fact, the question regarding the mere existence of such samplers was raised few years ago by Cormode, Muthukrishnan, and Rozenbaum in [9]. Last year in SODA 2010, Monemizadeh and Woodruff [22] answered this question affirmatively, however in an approximate sense. Before stating their results we give a formal definition of $L_{p}$-samplers.

Definition 1. Let $x \in \mathbb{R}^{n}$ be a non-zero vector. For $p>0$ we call the $L_{p}$ distribution corresponding to $x$ the distribution on $[n]$ that takes $i$ with probability

$$
\frac{\left|x_{i}\right|^{p}}{\|x\|_{p}^{p}}
$$

with $\|x\|_{p}=\left(\sum_{i=1}^{n}\left|x_{i}\right|^{p}\right)^{1 / p}$. For $p=0$, the $L_{0}$ distribution corresponding to $x$ is the uniform distribution over the nonzero coordinates of $x$.

We call a streaming algorithm a perfect $L_{p}$-sampler if it outputs an index according to this distribution and fails only if $x$ is the zero vector. An approximate $L_{p}$-sampler may fail but the distribution of its output should be close to the $L_{p}$ distribution. In particular, we speak of an $\epsilon$ relative error $L_{p}$-sampler if, conditioned on no failure, it outputs the index $i$ with probability $(1 \pm \epsilon)\left|x_{i}\right|^{p} /\|x\|_{p}^{p}+O\left(n^{-c}\right)$, where $c$ is an arbitrary constant. For $p=0$ the corresponding formula is $(1 \pm \epsilon) / k+O\left(n^{-c}\right)$, where $k$ is the number of non-zero coordinates in $x$. Unless stated otherwise we assume that the failure probability is at most $1 / 2$.

In this definition one can consider $c$ to be 2, but all existing constructions of $L_{p}$-samplers work for an arbitrary $c$ with just a constant factor increase in the space, so we will not specify $c$ in the following and ignore errors of probability $n^{-c}$.

\section{Previous work.}

A zero relative error $L_{0}$-sampler which uses $O\left(\log ^{3} n\right)$ bits was shown in [?]. In [22], the authors gave an $\epsilon$ relative error $L_{p}$-sampler for $p \in[0,2]$ which uses $\operatorname{poly}\left(\epsilon^{-1}, \log n\right)$ space. They also showed a 2-pass $O(\operatorname{polylog} n)$ space zero relative error $L_{p}$-sampler for any $p \in[0,2]$. In addition to these, they demonstrated that $L_{p}$-samplers can be used as a blackbox to obtain streaming algorithms for other problems such as $L_{p}$ estimation (for $p>2$ ), heavy hitters, and cascaded norms [14]. Unfortunately, due to the large exponents in their bounds, the $L_{p}$-samplers given there do not lead to efficient solutions for the aforementioned applications.

Very recently, Andoni, Krauthgamer and Onak in [1] improved the results of [22] considerably. Through the adaptation of a generic and simple method, named precision sampling, they managed to bring down the space upper bounds to $O\left(\frac{1}{\epsilon^{p}} \log ^{3} n\right)$ bits for $\epsilon$ relative error $L_{p^{-}}$-samplers for $p \in[1,2]$. Roughly speaking, the idea of precision sampling is to scale the input vector with random coefficients so that the $i$-th coordinate becomes the maximum with probability roughly proportional to $\left|x_{i}\right|^{p}$. Moreover the maximum (heavy) coordinate is found through a small-space heavy hitter algorithm. In more detail, the input vector $\left(x_{1}, \ldots, x_{n}\right)$ is scaled by random coefficients $\left(t_{1}^{-1}, \ldots, t_{n}^{-1}\right)$, where each $t_{i}$ is picked uniformly at random from $[0,1]$. Let $z=\left(x_{1} t_{1}^{-1}, \ldots, x_{n} t_{n}^{-1}\right)$ be the scaled vector. Here the important observation is $\operatorname{Pr}\left[t_{i}^{-1} \geq t\right]=1 / t$ and hence, for instance, by replacing $t$ with $\|x\|_{1} /\left|x_{i}\right|$, we get $\operatorname{Pr}\left[\left|z_{i}\right| \geq\right.$ $\left.\|x\|_{1}\right]=\left|x_{i}\right| /\|x\|_{1}$. (In the same manner, one can scale $x_{i}$ by $t_{i}^{-1 / p}$ instead of $t_{i}^{-1}$ and get a similar result for general $p$.) It turns out, we only need to we have a constant approximation to $\|x\|_{1}$ and look for a coordinate in $z$ that has reached a limit of $\Omega\left(\|x\|_{1}\right)$. On the other hand it is shown that the heaviest coordinate in $z$ has a weight of $\Omega\left(\log ^{-1} n\right)\|z\|_{1}$ (with constant probability), and thus a small-space heavy hitter computation can be used to find the maximum. In particular, the $L_{p}$-sampler of [1] adapts the popular count-sketch scheme [6] for this purpose.

\section{Our contributions.}

In this paper, we give $L_{p}$-samplers requiring only $O\left(\epsilon^{-p} \log ^{2} n\right)$ space for $p \in(1,2)$. For $p \in(0,1)$, our space bound is $O\left(\epsilon^{-1} \log ^{2} n\right)$, while for the $p=1$ case we have an $O(\log (1 / \epsilon)$ $\left.\epsilon^{-1} \log ^{2} n\right)$ space algorithm. In essence, our sampler follows the basic structure of the precision sampling method explained above. However compared to [1], we do a sharper analysis of the error terms in the count-sketch, and through additional ideas, we manage to get rid of a log factor and preserve the previous dependence on $\epsilon$. Roughly speaking, we use the fact that the error term in the count-sketch is bounded by the $L_{2}$ norm of the tail distribution of $z$ (the heavy coordinates do not contribute). On the other hand, taking the distribution of the random coefficients into account, we bound this by $O\left(\|x\|_{p}\right)$, which enables us to save a $\log$ factor. Additionally, to preserve the dependence on $\epsilon$, we have to use a slightly more powerful source of randomness for choosing the scaling factors (in contrast with the pairwise-independence of [1]), and take care of some subtle issues regarding the conditioning on the error terms which are not handled in the previous work (Lemma 3$).{ }^{1}$

As $p$ approaches zero, precision sampling becomes very inefficient, as the random coefficients $t_{i}^{-1 / p}$ tend to infinity. For the $p=0$ case, we present a zero relative error sampler through a completely different approach. Briefly, our $L_{0}$-sampler tries to detect a non-zero coordinate by picking random subsets of $[n]$. The non-zero coordinates are found by an exact sparse recovery procedure and Nisan's PRG [24] is applied to decrease the randomness involved. Our $O\left(\log ^{2} n\right)$ space bound compares favorably to the previous algorithms, which use respectively $O\left(\log ^{3} n\right)$ space [?] and poly $\left(\log n, \epsilon^{-1}\right)$ space [22] (the latter one gives only $\epsilon$ relative error sampling).

In Section 4, we prove that sampling from $0, \pm 1$ vectors requires $\Omega\left(\log ^{2} n\right)$ space, by a reduction from the communication complexity problem augmented indexing. In this special case $p$ is not relevant for $L_{p}$-sampling, hence this shows that our $L_{0}$-sampling algorithm uses the optimal space up to constant factors, and our $L_{p}$-sampler for $p \in(0,2)$ has the optimal space (up to constant factors) for $\epsilon>0$ a constant.

\footnotetext{
${ }^{1}$ Further we note that our algorithm not only produces a sample $i$ from the $L_{p}$ distribution, but also approximates $x_{i}$. Similar approximation is also produced by the $L_{p}$ sampler of [1], but they claim to give an approximation of $\left|x_{i}\right|^{p} /\|x\|_{p}^{p}$. However, this claim for $p<2$ cannot hold as it would contradict with the $\Omega\left(\epsilon^{-2}\right)$ space lower bound for estimating Hamming distance.
} 
Given a stream of length $n+1$ over the alphabet $[n]$, finding duplicates problem asks to output some $a \in[n]$ that has appeared at least twice in the stream. Observe that by the pigeon-hole principle, such $a$ always exists. Prior to our work, the best upper bound for finding duplicates was due to Gopalan and Radhakrishnan [13], who gave a one-pass $O\left(\log ^{3} n\right)$ bits randomized algorithm with constant failure rate. Here we settle the one-pass complexity of this problem by giving an $O\left(\log ^{2} n\right)$ space algorithm via a direct application of our $L_{1}$ sampler, and by giving an $\Omega\left(\log ^{2} n\right)$ lower bound afterwards. Combined with a sparse recovery procedure, our solution also generalizes to a near-optimal $O\left(\log ^{2} n+s \log n\right)$ space algorithm for finding duplicates in streams of length $n-s$, improving on the $O\left(s \log ^{3} n\right)$ result of [13].

Finally, we prove lower bounds for the problem of finding heavy hitters in update streams, which is closely related to the $L_{p}$-sampling problem. This lower bound is also obtained by a reduction from the augment indexing and proves that any $L_{p}$ heavy hitters algorithm (defined in Section 4.4) must use $\Omega\left(\frac{1}{\phi^{p}} \log ^{2} n\right)$ space, even in the strict turnstile model. Our lower bound essentially matches the known upper bounds $[8,6,15]$ which work in the general update model.

\section{Related work.}

In $[3,5]$, the authors have studied sampling from sliding windows, and the recent paper of Cormode et al. [10] generalizes the classical reservoir sampling to distributed streams. These works only consider insertion streams. The basic idea of random scaling used in [1] and in our paper has appeared earlier in the priority sampling technique $[11,7]$, where the focus is to estimate the weight of certain subsets of a vector, defined by a sequence of positive updates.

Finding duplicates in streams was first considered in the context of detecting fraud in click streams [20]. Muthukrishnan in [23] asked whether this problem can be solved in $O($ polylog $n)$ space using a constant number of passes. In [26], Tarui showed that any $k$-pass deterministic algorithm must use $\Omega\left(n^{\frac{1}{2 k-1}}\right)$ space to find a duplicate.

Heavy hitter algorithms have been studied extensively. The work of Berinde et al. [4] gives tight lower bounds for heavy hitters under insertion only streams. We are not aware of similar works on general update streams, although the recent works of $[2,27]$, where the authors show lower bounds for respectively approximate sparse recovery, and Johnson-Lindenstrauss transforms (via augmented indexing) is closely related.

\section{Notation.}

We write $[n]$ for the set $\{1, \ldots, n\}$. An update stream is a sequence of tuples $(i, u)$, where $i \in[n]$ and $u \in \mathbb{R}$. The stream of updates implicitly define an $n$-dimensional vector $x \in \mathbb{R}^{n}$ as follows. Initially, $x$ is the zero vector. An update of the form $(i, u)$ adds $u$ to the coordinate $x_{i}$ of $x$ (leaving the other coordinates unchanged). In the strict turnstile model we are guaranteed that all coordinates of $x$ are non-negative at the end of the stream (although negative updates are still allowed), in the general model such guarantee does not exist. Our algorithms (like most other algorithms in the literature) work by maintaining a linear sketch $L: \mathbb{R}^{n} \rightarrow \mathbb{R}^{m}$. When computing the space requirement of such a streaming algo- rithm, we assume all the updates are integers $(u \in \mathbb{Z})$ and the coordinates of the vector $x$ throughout the stream remain bounded by some value $M=\operatorname{poly}(n)$. We make sure that the matrix of $L$ has also polynomially bounded integer entries, this way maintaining $L(x)$ requires updating $m$ integer counters and requires $O(m \log n)$ bits with fast update time (especially since the matrices we consider are sparse). This discretization step is standard and thus we omit most details.

In the standard model for randomized streaming algorithms the random bits used (to generate the random linear map $L$, for example) are part of the space bound. In contrast, our lower bounds do not make any assumption on the working of the streaming algorithm and allow for the random oracle model, where the algorithm is allowed free access to a random string at any time. All lower bounds are proved through reductions from communication problems.

We say an event happens with low probability if the probability can be made less than $n^{-c}$. Here $c>0$ is an arbitrary constant, for example one can set $c=2$. The actual value of $c$ has limited effect on the space of our algorithm: it changes only the unspecified constants hidden in the $O$ notation. We will routinely ignore low probability events, sometime even $O(n)$ of them, which is okay as we leave $c$ unspecified. Events complementary to low probability events are referred to as high probability events.

For $0 \leq m \leq n$ we call the vector $x \in \mathbb{R}^{n} m$-sparse if all but at most $m$ coordinates of $x$ are zero. We define $\operatorname{Err}_{2}^{m}(x)=\min \|x-\hat{x}\|_{2}$, where $\hat{x} \in \mathbb{R}^{n}$ ranges over all the $m$-sparse vectors.

\section{THE $L_{P}$ SAMPLER}

In this section, we present our $L_{p}$ sampler algorithm. In the following, we assume $p \in(0,2)$. This particular method does not seem to be applicable for the $p=2$ case and we know of no $O\left(\log ^{2} n\right)$ space $L_{2}$-sampling algorithm. We treat the $p=0$ case separately later.

We start by stating the properties of the two streaming algorithms we are going to use. Both are based on maintaining $L(x)$ for a well chosen random linear map $L: \mathbb{R}^{n} \rightarrow \mathbb{R}^{n^{\prime}}$ with $n^{\prime}<n$.

The count-sketch algorithm [6] is so simple we cannot resist the temptation to define it here. For parameter $m$, the count-sketch algorithm works as follows. It selects independent samples $h_{j}:[n] \rightarrow[6 m]$ and $g_{j}:[n] \rightarrow\{1,-1\}$ from pairwise independent uniform hash families for $j \in[l]$ and $l=O(\log n)$. It computes the following linear function of $x$ for $j \in[l]$ and $k \in[6 m]: y_{k, j}=\sum_{i \in[n], h_{j}(i)=k} g_{j}(i) x_{i}$. Finally it outputs $x^{*} \in \mathbb{R}^{n}$ as an approximation of $x$ with $x_{i}^{*}=\operatorname{median}\left(g_{j}(i) y_{h(i), j}: j \in[l]\right)$ for $i \in[n]$.

The performance guarantee of the count-sketch algorithm is as follows. (For a compact proof see a recent survey by Gilbert and Indyk [12].)

Lemma 1. [6] For any $x \in \mathbb{R}^{n}$ and $m \geq 1$ we have $\mid x_{i}-$ $x_{i}^{*} \mid \leq \operatorname{Err}_{2}^{m}(x) / m^{1 / 2}$ for all $i \in[n]$ with high probability, where $x^{*}$ is the output of the count-sketch algorithm with parameter $m$. As a consequence we also have

$$
\operatorname{Err}_{2}^{m}(x) \leq\|x-\hat{x}\|_{2} \leq 10 \operatorname{Err}_{2}^{m}(x)
$$

with high probability, where $\hat{x}$ is the $m$-sparse vector best approximating $x^{*}$ (i.e., $\hat{x}_{i}=x_{i}^{*}$ for the $m$ coordinates $i$ with 
$\left|x_{i}^{*}\right|$ highest and is $\hat{x}_{i}=0$ for the remaining $n-m$ coordinates).

We will also need the following result for the estimation of $L_{p}$ norms.

Lemma 2. [?] For any $p \in(0,2]$ there is a streaming algorithm based on a random linear map $L: \mathbb{R}^{n} \rightarrow \mathbb{R}^{l}$ with $l=O(\log n)$ that outputs a value $r$ computed solely from $L(x)$ that satisfies $\|x\|_{p} \leq r \leq 2\|x\|_{p}$ with high probability.

Our streaming algorithm on Figure 1 makes use of a single count-sketch and two norm estimate algorithms. The countsketch is for the randomly scaled version $z$ of the vector $x$. One of the norm approximation algorithms is for $\|x\|_{p}$, the other one approximates $\operatorname{Err}_{2}^{m}(z)$ through the almost equal value $\|z-\hat{z}\|_{2}$. A standard $L_{2}$ approximation for $z$ works if we modify $z$ by subtracting $\hat{z}$ in the recovery stage. One can get arbitrary good approximations of $\operatorname{Err}_{2}^{m}(x)$ this way.

First we estimate the probability that the algorithm aborts because $s>\beta m^{1 / 2} r$. This depends on the scaling that resulted in $z$ and it will be important for us that the bound holds even after conditioning on any one scaling factor.

Lemma 3. Conditioned on an arbitrary fixed value $t$ of $t_{i}$ for a single index $i \in[n]$ we have $\operatorname{Pr}\left[s>\beta m^{1 / 2} r \mid t_{i}=t\right]=$ $O\left(\epsilon+n^{-c}\right)$.

Proof. First note that by Lemma 2 we have $r \geq\|x\|_{p}$ and $s \leq 2\|z-\hat{z}\|_{2}$ with high probability. By Lemma 1 we have $\|z-\hat{z}\| \leq 10 \operatorname{Err}_{2}^{m}(z)$ also with high probability. We may therefore assume that all of these inequalities hold, and in particular $r \geq\|x\|_{p}$ and $s \leq 20 \operatorname{Err}_{2}^{m}(z)$. It is therefore enough to bound the probability that $20 \operatorname{Err}_{2}^{m}(z)>$ $\beta m^{1 / 2}\|x\|_{p}$.

For simplicity (and without loss of generality) we assume that the fixed scalar is $t_{n}=t$ and will freely use $i$ for indexes in $[n-1]$.

Let $T=\beta\|x\|_{p}$. For each $i \in[n-1]$ we define two variables $z_{i}^{\prime}$ and $z_{i}^{\prime \prime}$ determined by $z_{i}$ as follows. The indicator variable $z_{i}^{\prime}=1$ if $\left|z_{i}\right|>T$ and 0 otherwise. We set $z_{i}^{\prime \prime}=z_{i}^{2}(1-$ $\left.z_{i}^{\prime}\right) / T^{2} \in[0,1]$. Let $S^{\prime}=\sum_{i \in[n-1]} z_{i}^{\prime}$ and $S^{\prime \prime}=\sum_{i \in[n-1]} z_{i}^{\prime \prime}$. Note that $T^{2} S^{\prime \prime}=\|z-w\|_{2}^{2}$, where $w$ is defined by $w_{i}=z_{i} z_{i}^{\prime}$ for $i \in[n-1]$ and $w_{n}=z_{n}$. Here $w$ is $\left(S^{\prime}+1\right)$-sparse, so we have $\operatorname{Err}_{2}^{m}(z) \leq T S^{\prime 1 / 2}$ unless $S^{\prime} \geq m$. It is therefore enough to bound the probabilities of the events $S^{\prime} \geq m$ and $S^{\prime \prime}>m \beta^{2}\|x\|_{p}^{2} /(20 T)^{2}=m / 400$, each with $O(\epsilon)$.

We have $\mathbb{E}\left[z_{i}^{\prime}\right]=\left|x_{i}\right|^{p} / T^{p}, \mathbb{E}\left[S^{\prime}\right] \leq \beta^{-p}=\epsilon^{1-p}$. By our choice of $m$ and the concentration of $S^{\prime}$ provided by $k$-wise independence we have $\operatorname{Pr}\left[S^{\prime} \geq m\right]=O(\epsilon)$ as needed. The calculation for $S^{\prime \prime}$ is similar. We have

$$
\mathbb{E}\left[z_{i}^{\prime \prime}\right]<\int_{\left|x_{i}\right|^{p} / T^{p}}^{\infty} x_{i}^{2} t^{-2 / p} T^{-2} d t=\frac{p}{2-p}\left|x_{i}\right|^{p} T^{-p} .
$$

Thus $\mathbb{E}\left[S^{\prime \prime}\right] \leq \frac{p}{2-p}\|x\|_{p}^{p} T^{-p}=O\left(\beta^{-p}\right)=O\left(\epsilon^{1-p}\right)$. Note that the $z_{i}^{\prime \prime}$ are not indicator variables as the $z_{i}^{\prime}$, but they are still $k$-wise independent random variables from $[0,1]$ and we can bound the probability of large deviation for $S^{\prime \prime}$ as we did for $S^{\prime}$. This completes the proof of the lemma.

The fact that our algorithm is an approximate $L_{p}$-sampler with both relative error and success probability $\Theta(\epsilon)$ follows from the following lemma. Indeed, if the probabilities were exactly $\epsilon\left|x_{i}\right|^{p} / r^{p}$ and if $\|x\|_{p} \leq r \leq 2\|x\|_{p}$ would always hold, we would make no relative error and the success probability would be $\mathbb{E}\left[\epsilon\|x\|_{p}^{p} / r^{p}\right] \geq \epsilon / 2^{p}$.
Lemma 4. The probability that the algorithm of Figure 1 outputs the index $i \in[n]$ conditioned on a fixed value for $r \geq\|x\|_{p}^{p}$ is $\left(\epsilon+O\left(\epsilon^{2}\right)\right)\left|x_{i}\right|^{p} / r^{p}+O\left(n^{-c}\right)$. The relative error of the estimate for $x_{i}$ is at most $\epsilon$ with high probability.

Proof. Optimally, we would output $i \in[n]$ if $\left|z_{i}\right|>$ $\epsilon^{-1 / p} r$. This happens if $t_{i}<\epsilon\left|x_{i}\right|^{p} / r^{p}$ and has probability exactly $\epsilon\left|x_{i}\right|^{p} / r^{p}$. We have to estimate the probability that something goes wrong and the algorithm outputs $i$ when this simple condition is not met or vice versa.

Three things can go wrong. First, if $s>m^{1 / 2} \beta r$ the algorithm fails. This is only a problem for our calculation if it should, in fact, output the index $i$. Lemma 3 bounds the conditional probability of this happening.

Having dealt with the $s>\beta m^{1 / 2} r$ case we may assume now $s \leq \beta m^{1 / 2} r$. We also make the assumptions (high probability by Lemma 2) that $\|z-\hat{z}\|_{2} \leq s$ and thus $\operatorname{Err}_{2}^{m}(z) \leq$ $\|z-\hat{z}\|_{2} \leq s \leq \beta m^{1 / 2} r$. Finally, we also assume $\left|z_{i}^{*}-z_{i}\right| \leq$ $\operatorname{Err}_{2}^{m}(z) / m^{1 / 2} \leq \beta r$ for all $i \in[n]$. This is satisfied with high probability by Lemma 1 .

A second source of error comes from this $\beta r$ possible difference between $z_{i}^{*}$ and $z_{i}$. This can only make a problem if $t_{i}$ is close to the threshold, namely $\left(\epsilon^{-1 / p}+\beta\right)^{-p}\left|x_{i}\right|^{p} / r^{p} \leq$ $t_{i} \leq\left(\epsilon^{-1 / p}-\beta\right)^{-p}\left|x_{i}\right|^{p} / r^{p}$. The probability of selecting $\bar{t}_{i}$ from this interval is $O\left(\beta / \epsilon^{1+1 / p}\left|x_{i}\right|^{p} / r^{p}\right)=O\left(\epsilon^{2}\left|x_{i}\right|^{p} / r^{p}\right)$ as required.

Finally, the third source of error comes from the possibility that $i$ should be output based on $\left|z_{i}\right|>\epsilon^{-1 / p} r$, yet we output another index $i^{\prime} \neq i$ because $z_{i^{\prime}}^{*} \geq z_{i}^{*}$. In this case we must have $t_{i^{\prime}}<\left(\epsilon^{-1 / p}-\beta\right)^{-p}\left|x_{i}\right|^{p} / r^{p}$. This has probability $O\left(\epsilon\left|x_{i^{\prime}}\right|^{p} / r^{p}\right)$. By the union bound the probability that such an index $i^{\prime}$ exists is $O\left(\epsilon\|x\|_{p}^{p} / r^{p}\right)=O(\epsilon)$. Pairwise independence is enough to conclude that the same bound holds after conditioning on $\left|z_{i}\right|>\epsilon^{-1 / p} r$. This finishes the proof of the first statement of the lemma.

The algorithm only outputs an index $i$ if $s \leq \beta m^{1 / 2} r$ and $\left|z_{i}^{*}\right| \leq \epsilon^{-1 / p} r$. The first implies that the absolute approximation error for $z_{i}$ is at most $\beta r$, while the second lower bounds the absolute value of the approximation itself by $\epsilon^{-1 / p} r$, thus ensuring a $\beta \epsilon^{1 / p}=\epsilon$ relative error approximation. Our approximation for $x_{i}=z_{i} t_{i}^{1 / p}$ is $z_{i}^{*} t^{1 / p}$, so the relative error is the same. Note that the inverse polynomial error probability comes from the various low probability events we neglected. The same is true for the additive error term in the distribution.

TheOREM 1. For $\delta>0$ and $\epsilon>0,0<p<2$ there is an $O(\epsilon)$ relative error one pass $L_{p}$-sampling algorithm with failing probability at most $\delta$ and having low probability that the relative error of the estimate for the selected coordinate is more than $\epsilon$. The algorithm uses $O_{p}\left(\epsilon^{-\max (1, p)} \log ^{2} n \log (1 / \delta)\right)$ space for $p \neq 1$ while for $p=1$ the space is $O\left(\epsilon^{-1} \log (1 / \epsilon) \log ^{2} n\right.$ $\log (1 / \delta))$.

Proof. Using Lemma 4 and the fact that $\|x\|_{p} \leq r \leq$ $2\|x\|_{p}$ with high probability one obtains that the failure probability of the algorithm in Figure 1 is at most $1-\epsilon / 2^{p}+$ $O\left(n^{-c}\right)$. Conditioning on obtaining an output, returning $i$ has probability $(1+O(\epsilon))\left|x_{i}\right|^{p} /\|x\|_{p}^{p}+O\left(n^{-c}\right)$. Clearly, the latter statement remains true for any number of repetitions and the failure probability is raised to power $v$ for $v$ repetitions. Thus using $v=O(\log (1 / \delta) / \epsilon)$ repetitions (taking the first non-failing output), the algorithm is an $O(\epsilon)$ relative error $\delta$ failure probability $L_{p}$-sampling algorithm. Here we 
Initialization Stage:

1. For $0<p<2, p \neq 1$ set $k=10\lceil 1 /|p-1|\rceil$ and $m=O\left(\epsilon^{-\max (0, p-1)}\right)$ with a large

enough constant factor.

2. For $p=1$ set $k=m=O(\log (1 / \epsilon))$ with a large enough constant factor.

3. Set $\beta=\epsilon^{1-1 / p}$ and $l=O(\log n)$ with a large enough constant factor.

4. Select $k$-wise independent uniform scaling factors $t_{i} \in[0,1]$ for $i \in[n]$.

5. Select the appropriate random linear functions for the execution of the count-sketch

algorithm and $L$ and $L^{\prime}$ for the norm estimations in the processing stage.

\section{Processing Stage:}

1. Use count-sketch with parameter $m$ for the scaled vector $z \in \mathbb{R}^{n}$ with $z_{i}=x_{i} / t_{i}^{1 / p}$.

2. Maintain a linear sketch $L(x)$ as needed for the $L_{p}$ norm approximation of $x$.

3. Maintain a linear sketch $L^{\prime}(z)$ as needed for the $L_{2}$ norm estimation of $z$.

\section{Recovery Stage:}

1. Compute the output $z^{*}$ of the count-sketch and its best $m$-sparse approximation $\hat{z}$.

2. Based on $L(x)$ compute a real $r$ with $\|x\|_{p} \leq r \leq 2\|x\|_{p}$.

3. Based on $L^{\prime}(z-\hat{z})$ compute a real $s$ with $\|z-\hat{z}\|_{2} \leq s \leq 2\|z-\hat{z}\|_{2}$.

4. Find $i$ with $\left|z_{i}^{*}\right|$ maximal.

5. If $s>\beta m^{1 / 2} r$ or $\left|z_{i}^{*}\right|<\epsilon^{-1 / p} r$ output FAIL.

6. Output $i$ as the sample and $z_{i}^{*} t_{i}^{1 / p}$ as an approximation for $x_{i}$.

Figure 1: Our approximate $L_{p}$-sampler with both success probability and relative error $\Theta(\epsilon)$

assume $v<n$ as otherwise recording the entire vector $x$ is more efficient.

The low probability of more than $\epsilon$ relative error in estimating $x_{i}$ also follows from Lemma 4 . In one round, the algorithm on Figure 1 uses $O(m \log n)$ counters for the countsketch and this dominates the counters for the norm estimators. Using standard discretization this can be turned into an $O\left(m \log ^{2} n\right)$ bit algorithm. For the discretization we also have to keep our scaling factors polynomial in $n$. Recall that in the continuous model these factors $t_{i}^{-1 / p}$ were unbounded. But we can safely declare failure if $t_{i}^{-1}>n^{c}$ for some $i \in[n]$ as this has low probability $n^{1-c}$. We have to do the $v$ repetitions of the algorithm in parallel to obtain a single pass streaming algorithm. This increases the space to $O\left(v m \log ^{2} n\right)$ which is the same as the one claimed in the theorem.

Note that the hidden constant in the space bound of the theorem depends on $p$, especially that $1 /(2-p), 1 / p$ and $1 /|1-p|$ factors come in. The last can always be replaced by a $\log (1 / \epsilon)$ factor but the former ones are harder to handle. For $p=2$ an extra $\log n$ factor seems to be necessary for an algorithm along these lines, see [1].

As we will see in Theorem 7, our space bound is tight for $\epsilon$ and $\delta$ constants. Note that the lower bound holds also if we only require the overall distribution of the $L_{p}$-sampler to be close to the $L_{p}$ distribution as opposed to the much more strict definition of $\epsilon$ relative error sampling.

\subsection{The $L_{0}$ Sampler}

For $p$ near zero, the method of precision sampling becomes intractable. This is because our scaling factors are $t_{i}^{-1 / p}$ which clearly rules out $p=0$. In the following we present a $L_{0}$ using a different approach. First we state the following well-known result on exact recovery of sparse vectors.

Lemma 5. Let $1 \leq s \leq n$. There is a choice $k=O(s)$ and random linear function $L: \mathbb{R}^{n} \rightarrow \mathbb{R}^{k}$ (generated from
$O(k \log n)$ random bits) and a recovery procedure that on input $L(x)$ outputs $x^{\prime} \in \mathbb{R}^{n}$ or DENSE such that for any $s$-sparse vector $x$ the output is $x^{\prime}=x$ with probability 1 and for any vector $x$ that is not s-sparse the output is DENSE with high probability.

THEOREM 2. There exists a zero relative error $L_{0}$ sampler which uses $O\left(\log ^{2} n \log (1 / \delta)\right)$ bits and outputs a coordinate $i \in[n]$ with probability at least $1-\delta$.

Proof. We first present our algorithm assuming a random oracle, and then we remove this assumption through the use of the pseudo-random generator of Nisan [24]. Let $I_{k}$ for $k=1, \ldots,\lfloor\log n\rfloor$ be subsets of $[n]$ of size $2^{k}$ chosen uniformly at random and $I_{0}=[n]$. For each $k$ we run the sparse recovery procedure of Lemma 5 on the vector $x$ restricted to the coordinates in $I_{k}$ with $s$ set to $\lceil 4 \log (1 / \delta)\rceil$. We return a uniform random non-zero coordinate from the first recovery that gives a non-zero $s$-sparse vector. The algorithm fails if each recovery algorithm returns zero or DENSE.

Let $J$ be the set of coordinates $i$ with $x_{i} \neq 0$ (the support of $x$ ). Disregarding the low probability error of the procedure in Lemma 5 this procedure returns each index $i \in J$ with equal probability and never returns an index outside $J$. To bound the failure probability we observe that for $|J| \leq s$ failure is not possible, while for $|J|>s$ one has $k \in[\lfloor\log n\rfloor]$ such that $\mathbb{E}\left[\left|I_{k} \cap J\right|\right]=2^{k}|J| / n$ is between $s / 3$ and $2 s / 3$. For this $k$ alone $1 \leq\left|I_{k} \cap J\right| \leq s$ is satisfied with probability at least $1-\delta$ by the Chernoff bound limiting failure probability by $\delta$.

To get rid of the random oracle we use Nisan's generator [24] that produces the random bits for the algorithm (including the ones describing $I_{k}$ and the ones for the eventual random choice from $\left.I_{k} \cap J\right)$ from an $O\left(\log ^{2} n\right)$ length seed. It fools every logspace tester including the one that tests for a fixed set $J \subseteq[n]$ and $i \in[n]$ if the algorithm (assuming correct reconstruction) would return $i$. Thus this version of the algorithm, now using $O\left(\log ^{2} n\right)$ random bits and 
$O\left(\log ^{2} \log (1 / \delta)\right)$ total space, is also a zero relative error $L_{0^{-}}$ sampler with failure probability bounded by $\delta+O\left(n^{-c}\right)$.

As we will see in Theorem 7, this space bound is also tight for $\delta$ a constant and better sampling is not possible even if we allow constant relative error or a small overall distance of the output from the $L_{0}$ distribution.

\section{FINDING DUPLICATES}

Recall that, given a data stream of length $n+1$ over the alphabet $[n]$, finding duplicates problem asks to output some $a \in[n]$ that has appeared at least twice in the stream.

Theorem 3. For any $\delta>0$ there is a $O\left(\log ^{2} n \log (1 / \delta)\right)$ space one-pass algorithm which, given a stream of length $n+$ 1 over the alphabet $[n]$, outputs an $i \in[n]$ or FAIL, such that the probability of outputting FAIL is at most $\delta$ and the algorithm outputs a letter $i \in[n]$ that is no duplicate with low probability.

Proof. Let $x$ be an $n$-dimensional vector, initially zero at each coordinate. We run the $L_{1}$-sampler of Theorem 1 on $x$, with both relative error and failure probability set to $1 / 2$. Before we start processing the stream, we subtract 1 from each coordinate of $x$; i.e., we feed the updates $(i,-1)$ for $i=1, \ldots n$ to the $L_{1}$ sampling algorithm. When a stream item $i \in[n]$ comes, we increase $x_{i}$ by 1 ; i.e., we generate the update $(i, 1)$.

Observe that when the stream is exhausted, we have $x_{i} \geq$ 1 for items $i$ that have at least two occurrences in the stream, $x_{i}=0$ for items that appear once, and $x_{i}=-1$ for items that do not appear. Note that our $L_{1}$-sampler, if it does not fail, outputs an index $i$ and an approximation $x^{*}$ of $x_{i}$. If $x^{*}$ is positive, we output $i$, if it is negative or the $L_{1}$-sampler fails, we output FAIL. We have $\sum_{i=1}^{n} x_{i}=1$, hence a perfect $L_{1}$ sample from $x$ is positive with more than half probability. Taking into account that our $L_{1}$-sampler has $1 / 2$ relative error and failure probability (and neglecting for a second the chance that $x^{*}$ has different sign from $x_{i}$ ) we conclude that we output a duplicate with probability at least $1 / 4$. The event that $x^{*}$ does not have the same sign as $x_{i}$ (and thus the relative error is at least 1 ) has low probability. This low probability can increase the failure probability and/or introduce error when we output non-duplicate items.

Repeating the algorithm $O(\log (1 / \delta))$ times in parallel and accepting the first non-failing output reduces the failure rate to $\delta$ but keeps the error rate low.

As we will see in Theorem 6, our space bound is tight for $\delta<1$ a constant.

It is natural to study the duplicates problem for other ranges of parameters. Assume that we have a stream of length $n-s \leq n$ over the alphabet $[n]$. For this problem, Gopalan et al. [13] gave an $O\left((s+1) \log ^{3} n\right)$ bits algorithm and an $\Omega(s)$ lower bound. Here we give an algorithm which uses $O\left(s \log n+\log ^{2} n\right)$ space.

TheOREM 4. For any $\delta>0$ there is an $O\left(\log ^{2} n \log 1 / \delta+\right.$ $s \log n)$ space one-pass algorithm which, given a stream of length $n-s$ over the alphabet [n], outputs NO-DUPLICATE with probability 1 if the input sequence has no duplicates, otherwise it outputs $i \in[n]$ or reports FAIL. The returned number is a duplicate with high probability while the probability of returning FAIL is at most $\delta$.
Proof. Let $x$ be an $n$-dimensional vector updated according to the description in the proof of Theorem 3; i.e., $x_{i}$ is one less than the number of times $i$ appears in the stream. In parallel, we run the exact recovery procedure from Lemma 5 with parameter $5 s$ and the $1 / 2$ relative error $L_{1}$-sampler of Theorem 1 with failure rate $1 / 2$, both on the vector $x$. If the recovery algorithm returns a vector (as opposed to DENSE) we proceed and give the correct output assuming we have learned the entire $x$. Otherwise we consider the output of the sampling algorithm. If it is $\left(i, x^{*}\right)$ with $x^{*}>0$ we report $i$ as a duplicate otherwise (if $x^{*} \leq 0$ or the sampling algorithm fails) we output FAIL. Define

$$
\|x\|_{1}^{+}=\sum_{i: x_{i}>0}\left|x_{i}\right| \quad \text { and } \quad\|x\|_{1}^{-}=\sum_{i: x_{i}<0}\left|x_{i}\right| .
$$

Note that $\|x\|_{1}^{+}-\|x\|_{1}^{-}=\sum_{i=1}^{n} x_{i}=-s$. If $\|x\|_{1}^{+}+\|x\|_{1}^{-} \leq$ $5 s$, then $x$ is $5 s$-sparse, thus the sparse recovery procedure outputs $x$ and the algorithm makes no error. Note that the no repetition case falls into this category. If, however, $\|x\|_{1}^{+}+\|x\|_{1}^{-}>5 s$, then the probability that a perfect $L_{1}$ sample from $x$ is positive is $\|x\|_{1}^{+} /\|x\|_{1}>2 / 5$. Taking into account the relative error and failing probability (but ignoring the low probability event of the sampler outputting a wrong sign or sparse recovery algorithm reporting a vector), we conclude that with probability at least $1 / 10$ we get a positive sample and a correct output, otherwise we output FAIL. The failure probability can be decreased to $\delta$ by $O(\log (1 / \delta))$ independent repetitions of the sampler. Note that the sparse recovery does not have to be repeated as it has low error probability.

The sparse recovery procedure takes $O(s \log n)$ bits by Lemma 5 for $s>0$ (it takes $O(\log n$ ) bits for $s=0$ ) and each instance of the $L_{1}$-sampler requires $O\left(\log ^{2} n\right)$ bits by Theorem 4, totaling $O\left(s \log n+\log ^{2} n \log 1 / \delta\right)$ bits.

Here we do not have a matching lower bound, but only the $\Omega\left(\log ^{2} n+s\right)$ that follows from the $\Omega(s)$ bound in [13] and our $\Omega\left(\log ^{2} n\right)$ bound on the original version of the duplicates problem.

We remark the last two theorems can be stated in a bit more general form. Instead of considering repetitions in data streams one can consider the problem of finding an index $i$ with $x_{i}>0$ for a vector $x \in \mathbb{Z}^{n}$ given by an update stream. Let $s=-\sum_{i=1}^{n} x_{i}$. If $s<0$, then a positive coordinate exists and the algorithm of Theorem 3 finds one using $O\left(\log ^{2} n \log (1 / \delta)\right)$ space with low error and at most $\delta$ failure probability. If $s \geq 0$ a positive coordinate does not necessarily exist, but the algorithm of Theorem 4 finds one, report none exists or fails with the error, failure and space bounds claimed there.

Let us consider finally the version of the duplicates problem, where we have a stream of length $n+s>n$ over the alphabet $[n]$. Our lower and upper bounds are even farther in this case. A duplicate can be found using $O\left(\min \left\{\log ^{2} n,(n / s) \log n\right\}\right)$ bits of memory in one pass with constant probability as follows. If we sample a random item from the stream, it will appear again unless that was the last appearance of the letter. As there are at most $n$ last appearances in the stream of length $n+s$, the probability for a uniform random sample to repeat later is at least $s /(n+s)$. Therefore, if $n / s<\log n$, we can sample $4\lceil n / s\rceil$ items from the stream uniformly at random and check if one of them appears again to obtain 
a constant error algorithm for finding duplicates. If on the other hand $n / s \geq \log n$, we use the algorithm in Theorem 3 .

Combining our lower bound for the original version of the duplicates problem with the simple lower bound of $\Omega(\log n)$, we conclude that any streaming algorithm that finds a duplicate in length $n+s$ streams must use $\Omega\left(\log ^{2}(n / s)+\log n\right)$ bits.

\section{LOWER BOUNDS}

All our lower bounds follow from the augmented indexing problem. This problem is defined as follows. Let $k$ and $m$ be positive integers. The first player Alice, is given a string $x \in[k]^{m}$, while the second player Bob is given an integer $i \in[m]$ and $x_{j}$ for $j<i$. Alice sends a single message to Bob and Bob should output $x_{i}$.

Lemma 6. [21] In any one-way protocol in the joint random source model with success probability at least $1-\delta>$ $\frac{3}{2 k}$, Alice must send a message of size $\Omega((1-\delta) m \log k)$.

We will use this lemma by reducing augmented indexing to other communication or streaming problems.

\subsection{Universal Relation}

Consider the following two player communication game. Alice gets a string $x \in\{0,1\}^{n}$, Bob gets $y \in\{0,1\}^{n}$ with the promise that $x \neq y$. The players exchange messages and the last player to receive a message must output an index $i \in[n]$ such that $x_{i} \neq y_{i}$. We call this the universal relation communication problem and denote it by $\mathrm{UR}^{\mathrm{n}}$.

This relation has been studied in detail for deterministic communication, as it naturally arises in the context of Karchmer-Wigderson games [18]. We note however that our definition is slightly unusual: in most settings both players must obtain the same index $i$ such that $x_{i} \neq y_{i}$, whereas we are satisfied with the last player to receive a message learning such an $i$. Clearly, the stronger requirement can be met in $\lceil\log n\rceil$ additional bits and one additional round. The additional bits are needed in deterministic case but we are not concerned with $O(\log n)$ terms for our bounds, so the two models are almost equivalent up to the shift of one in the number of rounds.

The best deterministic protocol for $\mathrm{UR}^{\mathrm{n}}$ is due to Tardos and Zwick [25]. Improving a previous result by Karchmer [17], they gave a 3 round deterministic protocol using $n+2$ bits of communication with both players learning the same index $i$ and showed that $n+1$ bits is necessary for such a protocol. They also gave an $n-\lfloor\log n\rfloor+2$ bit 2 round deterministic protocol for our weaker version of the problem, which is also tight except for the +2 term. They also gave an $n-\lfloor\log n\rfloor+4$ bit 4 round protocol, where both players find an index where $x$ and $y$ differ - but not necessarily the same index. This shows that finding the same difference is harder.

Let $R_{\delta}^{k}(U)$ denote the $k$-round $\delta$-error communication complexity of the communication problem $U$. We write $R_{\delta}(U)$ to denote the $\delta$-error communication complexity when the number of rounds is not bounded. The next proposition follows from similar ideas that were used in Theorem 2.

Proposition 1. It holds that $R_{\delta}^{1}\left(\mathrm{UR}^{\mathrm{n}}\right)=O\left(\log ^{2} n \log \frac{1}{\delta}\right)$ and $R_{\delta}^{2}\left(\mathrm{UR}^{\mathrm{n}}\right)=O\left(\log n \log \frac{1}{\delta}\right)$.
Proof (SKETCh). One way to deduce the one round protocol is from Theorem 2. Alice and Bob run a single pass $L_{0}$-sampling algorithm on $x-y$. This can be achieved by a single message from Alice to Bob containing the memory after the first set of updates as in the proof of Theorem 8. The sample $i$ Bob finds is an (almost uniform random) index with $x_{i} \neq y_{i}$.

A closer look shows that the algorithm of Theorem 2 makes $\lceil\log n\rceil+1$ guesses for the number of non-zero coordinates of $x-y$ and picks a random subset $I \subseteq[n]$ of proper size for each such guess. If the players are allowed two rounds, the number of bits sent can be reduced to $O\left(\log n \log \delta^{-1}\right)$ as follows. In the first round, the players estimate the number of non-zero coordinates of $x-y$ up to a factor of 2 via a standard Hamming distance estimation protocol (this takes $O\left(\log n \log \delta^{-1}\right)$ bits $)$. In the second round, Bob picks a random subset of $I$ of proper size and sends a recovery matrix about $I$ of size $O\left(\log n \log \delta^{-1}\right)$ to Alice.

We remark that along similar lines one can find an $\tilde{O}(\log n \log 1 / \delta)$ space two-pass zero relative error $L_{0}$-sampling algorithm, by estimating $L_{0}$ of the vector defined by the stream in the first pass using [16]. Next we will show that the above proposition is best possible up to the $O\left(\log \delta^{-1}\right)$ terms. We start with an averaging lemma.

Lemma 7. Any protocol for $\mathrm{UR}^{\mathrm{n}}$ can be turned into one that outputs every index $i \in[n]$ with $x_{i} \neq y_{i}$ with the same probability. The new protocol uses a joint random source. The number of bits sent, the number of rounds and the error probability does not change.

PROOF. Using the joint random source the players take a uniform random permutation $\pi$ of $[n]$ and use it to permute the digits of $x$ and $y$. Further they take a uniform random subset $S \subseteq[n]$ and flip the digits with coordinates in $S$. This requires no communication. Then they run the original protocol on the modified inputs and report $\pi^{-1}(i)$ if the original protocol reports $i$. All indices where $x$ and $y$ differ are reported with equal probability by symmetry.

THEOREM 5. For any $\delta<1$ constant we have $R_{\delta}^{1}\left(\mathrm{UR}^{\mathrm{n}}\right)=$ $\Omega\left(\log ^{2} n\right)$ and $R_{\delta}=\Omega(\log n)$.

Proof. The second bound comes from considering a uniform random pair $(x, y)$ with Hamming distance 1. Either player needs to get $\log n$ bits of information to learn the only index where the strings differ.

To prove the first bound suppose Alice and Bob wants to solve the augmented indexing problem with Alice receiving $z \in\left[2^{t}\right]^{s}$ and Bob getting $i \in[s]$ and $z_{j}$ for $j<i$.

Let them construct real vectors $u$ and $v$ as follows. Let $e_{q} \in \mathbb{R}^{2^{t}}$ be the standard unit vector in the direction of coordinate $q$. Alice forms the vectors $w_{j}$ by concatenating $2^{s-j}$ copies of $e_{z_{j}}$, then she forms $u$ by concatenating these vectors $w_{j}$ for $j \in[s]$. The dimension of $u$ is $n=\left(2^{s}-1\right) 2^{t}$. Bob obtains $v$ by concatenating the same vectors $w_{j}$ for $j \in[i-1]$ (these are known to him) and then concatenating enough zeros to reach the same dimension $n$.

Now Alice and Bob perform the $R_{\delta}^{1}\left(\mathrm{UR}^{\mathrm{n}}\right)$ length $\delta$ error one round protocol for $\mathrm{UR}^{\mathrm{n}}$. By Lemma 7 we may assume the protocol returns a uniform random index where $u$ and $v$ differ. Note that each such index reveals one coordinate $z_{j} \in$ $\left[2^{t}\right]$ to Bob for $j \geq i$. As $z_{j}$ is revealed by $2^{s-j}$ such indices more than half the time when the $\mathrm{UR}^{\mathrm{n}}$ protocol does not err 
Bob learns the correct value of $z_{i}$. This yields a $R_{\delta}^{1}\left(\mathrm{UR}^{\mathrm{n}}\right)$ length one way protocol for augmented indexing with error probability $(1+\delta) / 2$. By Lemma 6 we have $R_{\delta}^{1}\left(\mathrm{UR}^{\mathrm{n}}\right)=$ $\Omega(s t)$. Choosing $s=t$ proves the theorem.

\subsection{Finding Duplicates}

THEOREM 6. Any one-pass streaming algorithm that outputs a duplicate with constant probability uses $\Omega\left(\log ^{2} n\right)$ space. This remains true even if the stream is not allowed to have an element repeated more than twice.

Proof. We show our claim by a reduction from the universal relation. Each of Alice and Bob is given a binary string of length $n$, respectively $x$ and $y$. Further, the players are guaranteed that $x \neq y$. Alice sends a message to Bob, after which Bob must output an index $i \in[n]$ such that $x_{i} \neq y_{i}$. By Theorem 5, to solve this problem with $1 / 2$ error probability requires $\Omega\left(\log ^{2} n\right)$ bits for one-way communication. Alice constructs the set $S=\left\{2 i-1+x_{i} \mid i \in[n]\right\} \subseteq[2 n]$ and Bob constructs $T=\left\{2 i-y_{i} \mid i \in[n]\right\} \subseteq[2 n]$. Observe that $|S|=|T|=n$ and $x_{i} \neq y_{i}$ if and only if either $2 i$ or $2 i-1$ is in both $S$ and $T$.

Next, using the shared randomness, players pick a random subset $P$ of $[2 n]$ of size $n$. We have

$$
\operatorname{Pr}[|S \cap P|+|T \cap P| \geq n+1]>1 / 8 .
$$

To see this let $i \in S \cap T$ and $j \in[2 n] \backslash(S \cap T)$. We have $|P \cap\{i, j\}|=1$ with probability more than $1 / 2$. The sets $P$ satisfying this can be partitioned into classes of size four by putting $Q \cup\{i\}, Q \cup\{j\}$ and their complements in the same class for any $Q \subseteq[2 n] \backslash\{i, j\},|Q|=n-1$. Clearly, at least one of the four sets $P$ in each class satisfies $|S \cap P|+|T \cap P|>$ $n$.

Given a streaming algorithm $A$ for finding duplicates, Alice feeds the elements of $S \cap P$ to $A$ and sends the memory contents over to Bob, along with the integer $|S \cap P|$. If $|S \cap P|+|T \cap P|<n+1$, Bob outputs FAIL. Otherwise, feeds arbitrary $n+1-|S \cap P|$ elements of $T \cap P$ to $A$. Note that no element repeats more than twice.

On the other hand $|P|=n$ and we always give $n+1$ elements of $P$ to the algorithm. Also with constant probability, Bob finds an $a \in S \cap T$, which in turn reveals an $i$ such that $x_{i} \neq y_{i}$. Therefore by Theorem 5 , any algorithm for finding duplicates must use $\Omega\left(\log ^{2} n\right)$ bits.

\section{3 $\quad L_{p}$-sampling}

Our algorithm for the duplicates problem (Theorem 3) is based on $L_{1}$-sampling, thus the matching lower bound for the duplicates problem implies a similar bound for $L_{1}$ sampling. Here we show an $\Omega\left(\log ^{2} n\right)$ lower bound for $L_{p^{-}}$ sampling for all $p$. Notice that the $L_{p}$ distribution corresponding to $0, \pm 1$ vectors are independent of $p$, so $p$ does not have to be specified for the next theorem.

THEOREM 7. Any one pass $L_{p}$-sampler with an output distribution, whose variation distance from the $L_{p}$ distribution corresponding to $x$ is at most $1 / 3$, requires $\Omega\left(\log ^{2} n\right)$ bits of memory. This holds even when all the coodinates of $x$ are guaranteed to be $-1,0$ or 1 .

For constants $\delta<1$ and $\epsilon<1$ the same lower bound holds for any $\epsilon$ relative error $L_{p}$-sampler with failure probability $\delta$.

Proof. Consider the $L_{1}$ sampling algorithm that we used to prove Theorem 3. Given a stream of items from $[n]$ we turned it to an update stream for an $n$ dimensional vector $x$ by first producing an update $(i,-1)$ for all $i \in[n]$ and then for any letter $i$ in the stream producing an update $(i, 1)$. Assuming that no item appears more than twice in the stream all coordinates of the final vector $x$ are $-1,0$ or 1 . The $L_{1}$ distribution for $x$ puts weight more than $1 / 2$ on the coordinates having value 1 . These are the duplicates. Thus if we have another algorithm such that the variation distance of its output is at most $1 / 3$ from this $L_{1}$ distribution, then it returns a coordinate with value 1 with probability at least $1 / 6$. For an $\epsilon$ relative error $\delta$ failure probability approximate $L_{p}$-sampler the same probability is at least $(1-\epsilon)(1-\delta)-$ $n^{1-c}$. Finding a coordinate in $x$ with value 1 is the same as finding a duplicate in the original stream, so we need $\Omega\left(\log ^{2} n\right)$ memory by Theorem 6 .

\subsection{Heavy Hitters}

The heavy hitters problem in the streaming model is defined as follows. Let $x$ be an $n$-dimensional integer vector given by an update stream. A heavy hitters algorithm with parameters $p>0$ and $\phi>0$ is required to output a set $S \subseteq[n]$ that contains all $i$ with $\left|x_{i}\right| \geq \phi\|x\|_{p}$ and no $i$ such that $\left|x_{i}\right| \leq \frac{\phi}{2}\|x\|_{p}$. We call such $S$ a valid heavy hitter set. ${ }^{2}$ In this part, we show a tight lower bound for the space complexity of randomized algorithms (assuming constant probability of error) for the heavy hitter problem. First we briefly review the upper bounds.

The count-median algorithm from [8] gives a $O\left(\phi^{-1} \log ^{2} n\right)$ space upper bound for the case of $p=1$. Here we note the count-sketch [6] in fact gives a $O\left(\phi^{-p} \log ^{2} n\right)$ space upper bound for all $p \in(0,2]$. The case of $p=2$ easily follows from Lemma 1. Let $d=\operatorname{Err}_{2}^{m}(x) / m^{1 / 2}$. In general it holds $d \leq\|x\|_{p} / m^{1 / p}$ for any $p \in(0,2]$. Indeed, let $H \subset[n]$ be the set of indices for which $d^{2}=\sum_{i \in H} x_{i}^{2} / m$ and let $c=\max _{i \in H}\left|x_{i}\right|$. Then we have $\|x\|_{p}^{p} / m=\sum_{i \in[n]}\left|x_{i}\right|^{p} / m \geq$ $c^{p}+\sum_{i \in H}\left|x_{i}\right|^{p} / m \geq c^{p}+c^{p-2} \sum_{i \in H} x_{i}^{2} / m=c^{p}+c^{p-2} d^{2} \geq$ $c^{p}\left((1-p / 2)+(p / 2) c^{-2} d^{2} \geq c^{p}\left(c^{-2} d^{2}\right)^{p / 2}=d^{p}\right.$. Therefore setting $m=1 / \phi^{p}$ in the count-sketch scheme gives the desired result

We remark that a similar upper bound for the heavy hitter problem is shown in [15] (cf. Theorem 1), albeit via different arguments. In the next theorem, we show that the above upper bound is tight for any reasonable range of parameters. Our lower bound holds even in the strict turnstile model and even for very short streams.

Theorem 8. Let $p>0$ and $\phi \in(0,1)$ be a reals. Any one pass heavy hitter algorithm in the strict turnstile model uses $\Omega\left(\phi^{-p} \log ^{2} n\right)$.

Proof. Suppose there is a one pass heavy hitter algorithm for parameters $p$ and $\phi$. We allow for a random oracle and assume the updates are polynomially bounded in $n$ and integers. We can also restrict the number of updates to be $O\left(\phi^{-p} \log n\right)$ and assume all coordinates of the final vector are positive (strict turnstile model). We turn this streaming algorithm into a protocol for augmented indexing in a similar way as we transformed the protocol for $\mathrm{UR}^{\mathrm{n}}$ to a protocol for augmented indexing in the proof of Theorem 5 .

\footnotetext{
${ }^{2}$ In general, the parameter $\frac{1}{2} \phi$ can be replaced by any $\epsilon<\phi$. Since here our focus is on lower bounds, we have simplified the definition.
} 
The exponential growth is now achieved not by repetition but by multiplying the coordinates with a growing factor.

Suppose Alice and Bob wants to solve the augmented indexing problem and Alice receives $y \in\left[2^{t}\right]^{s}$ and Bob gets $i \in[s]$ and $y_{j}$ for $j<i$. Let them construct real vectors $u$ and $v$ as follows. Let $b=\left(1-(2 \phi)^{p}\right)^{-1 / p}$ and let $e_{q} \in \mathbb{R}^{2^{t}}$ be the standard unit vector in the direction of coordinate $q$. Alice obtains $u$ by concatenating the vectors $\left\lceil b^{s-j}\right\rceil e_{y_{j}}$ for $j \in[s]$. The dimension of $u$ is $n^{\prime}=s 2^{t}$. Bob obtains $v$ by concatenating the same vectors for $j \in[i-1]$ and then concatenating enough zeros, namely $(s-i+1) 2^{t}$, to reach the same dimension $n^{\prime}$. Now Alice and Bob perform the heavy hitter algorithm for the vector $x=u-v$ as follows. Alice generates the necessary updates to increase the initially zero vector $x \in \mathbb{Z}^{n}$ to reach $x=u$, maintains the memory content throughout these updates and sends the final content to Bob. Now Bob generates the necessary updates to decrease $x=u$ to its final value $x=u-v$ and maintains the memory throughout. Finally Bob learns the heavy hitter set $S$ the streaming algorithm produces and outputs $z \in\left[2^{t}\right]$ if the smallest index in $S$ is $(i-1) 2^{t}+z$.

We claim that the above protocol errs only if the streaming algorithm makes an error. Notice that all coordinates of $x_{l}$ of $x=u-v$ are zero except the ones of the form $x_{l_{j}}=\left\lceil b^{s-j}\right\rceil$ for $l_{j}=(j-1) 2^{t}+y_{j}$, where $i \leq j \leq s$. Thus $x_{l_{i}}$ is the first non-zero coordinate. So the claim is true if $x_{l_{i}} \geq \phi\|x\|_{p}$. Using $\lceil v\rceil<2 v$ for $v \geq 1$ we get exactly this:

$$
\begin{aligned}
\phi^{p}\|x\|_{p}^{p} & =\phi^{p} \sum_{j=i}^{s}\left\lceil b^{s-j}\right\rceil^{p} \\
& <(2 \phi)^{p} b^{p(s-i+1)} /\left(b^{p}-1\right) \\
& =b^{p(s-i)} \quad\left(\text { since } b^{p}=1 /\left(1-(2 \phi)^{p}\right)\right) \\
& \leq x_{l_{i}}^{p}
\end{aligned}
$$

Let us now choose $s=\left\lceil(2 \phi)^{-p} \log n\right\rceil$ and $t=\lceil\log n / 2\rceil$. For large enough $n$ this gives $n^{\prime}=s 2^{t}<n$ and all coordinates of $x$ throughout the procedure remain under $n$. Still if the streaming algorithm works with probability over $1 / 2$, then by Lemma 6 the message size of the devised protocol is $\Omega(s t)=\Omega\left(\phi^{-p} \log ^{2} n\right)$. This proves the theorem as the message size of the protocol is the same as the memory size of the streaming algorithm.

\section{REFERENCES}

[1] Alex Andoni, Robert Krauthgamer, and Krzysztof Onak. Streaming algorithms via precision sampling. Manuscript, 2010.

[2] Khanh Do Ba, Piotr Indyk, Eric Price, and David P. Woodruff. Lower bounds for sparse recovery. In SODA, pages 1190-1197, 2010.

[3] Brian Babcock, Mayur Datar, and Rajeev Motwani. Sampling from a moving window over streaming data. In SODA, pages 633-634, 2002.

[4] Radu Berinde, Graham Cormode, Piotr Indyk, and Martin J. Strauss. Space-optimal heavy hitters with strong error bounds. In PODS, pages 157-166, 2009.

[5] Vladimir Braverman, Rafail Ostrovsky, and Carlo Zaniolo. Optimal sampling from sliding windows. In PODS, pages 147-156, 2009.

[6] Moses Charikar, Kevin Chen, and Martin
Farach-Colton. Finding frequent items in data streams. Theor. Comput. Sci., 312(1):3-15, 2004.

[7] Edith Cohen, Nick G. Duffield, Haim Kaplan, Carsten Lund, and Mikkel Thorup. Stream sampling for variance-optimal estimation of subset sums. In $S O D A$, pages 1255-1264, 2009.

[8] Graham Cormode and S. Muthukrishnan. An improved data stream summary: the count-min sketch and its applications. J. Algorithms, 55(1):58-75, 2005.

[9] Graham Cormode, S. Muthukrishnan, and Irina Rozenbaum. Summarizing and mining inverse distributions on data streams via dynamic inverse sampling. In $V L D B$, pages 25-36, 2005.

[10] Graham Cormode, S. Muthukrishnan, Ke Yi, and Qin Zhang. Optimal sampling from distributed streams. In PODS, pages 77-86, 2010.

[11] Nick G. Duffield, Carsten Lund, and Mikkel Thorup. Priority sampling for estimation of arbitrary subset sums. J. ACM, 54(6), 2007.

[12] Anna Gilbert and Piotr Indyk. Sparse recovery using sparse matrices. In Proceeding of IEEE, 2010.

[13] Parikshit Gopalan and Jaikumar Radhakrishnan. Finding duplicates in a data stream. In Proceedings of the twentieth Annual ACM-SIAM Symposium on Discrete Algorithms, SODA '09, pages 402-411, Philadelphia, PA, USA, 2009. Society for Industrial and Applied Mathematics.

[14] T. S. Jayram and David P. Woodruff. The data stream space complexity of cascaded norms. In FOCS, pages 765-774, 2009.

[15] Daniel M. Kane, Jelani Nelson, Ely Porat, and Woodruff David P. Fast moment estimation in data streams in optimal space. Manuscript, 2010.

[16] Daniel M. Kane, Jelani Nelson, and David P. Woodruff. An optimal algorithm for the distinct elements problem. In Proceedings of the twenty-ninth ACM SIGMOD-SIGACT-SIGART symposium on Principles of database systems of data, PODS '10, pages 41-52, New York, NY, USA, 2010. ACM.

[17] Mauricio Karchmer. A New Approach to Circuit Depth. PhD thesis, MIT, 1989.

[18] Mauricio Karchmer and Avi Wigderson. Monotone circuits for connectivity require super-logarithmic depth. In Proceedings of the twentieth annual ACM symposium on Theory of computing, STOC '88, pages 539-550, New York, NY, USA, 1988. ACM.

[19] Donald E. Knuth. The Art of Computer Programming, Volume II: Seminumerical Algorithms. Addison-Wesley, 1969.

[20] Ahmed Metwally, Divyakant Agrawal, and Amr El Abbadi. Duplicate detection in click streams. In $W W W$, pages 12-21, 2005.

[21] Peter Bro Miltersen, Noam Nisan, Shmuel Safra, and Avi Wigderson. On data structures and asymmetric communication complexity. In Proceedings of the twenty-seventh annual ACM symposium on Theory of computing, STOC '95, pages 103-111, New York, NY, USA, 1995. ACM.

[22] Morteza Monemizadeh and David P. Woodruff. 1-pass relative-error $\mathrm{l}$-sampling with applications. In $S O D A$, pages 1143-1160, 2010. 
[23] S. Muthukrishnan. Data Streams: Algorithms and Applications.

[24] N. Nisan. Pseudorandom generators for space-bounded computations. In Proceedings of the twenty-second annual ACM symposium on Theory of computing, STOC '90, pages 204-212, New York, NY, USA, 1990. ACM.

[25] Gabor Tardos and Uri Zwick. The communication complexity of the universal relation. In Proceedings of the 12th Annual IEEE Conference on Computational Complexity, pages 247-, Washington, DC, USA, 1997. IEEE Computer Society.

[26] Jun Tarui. Finding a duplicate and a missing item in a stream. In Jin-Yi Cai, S. Cooper, and Hong Zhu, editors, Theory and Applications of Models of Computation, volume 4484 of Lecture Notes in Computer Science, pages 128-135. Springer Berlin / Heidelberg, 2007.

[27] David Woodruff and T. S. Jayram. Optimal bounds for johnson-lindenstrauss transforms and streaming problems with low error. In SODA, 2011. 